QUARTERLY OF APPLIED MATHEMATICS

VOLUME LXIX, NUMBER 3

SEPTEMBER 2011, PAGES 425-443

S 0033-569X(2011)01207-3

Article electronically published on April 5, 2011

\title{
DELTA SHOCK WAVES FOR THE CHROMATOGRAPHY EQUATIONS AS SELF-SIMILAR VISCOSITY LIMITS
}

\author{
BY
}

MEINA SUN

School of Mathematics and Information, Ludong University, Yantai 264025, People's Republic of China and Laboratory of Mathematics Physics, Wuhan Institute of Physics and Mathematics, The Chinese Academy of Sciences, Wuhan 430071, People's Republic of China

Abstract. The Riemann problem for the changed form of the chromatography system is considered here. It can be shown that the delta shock wave appears in the Riemann solution for exactly specified initial states. The generalized Rankine-Hugoniot relation of the delta shock wave is derived in detail. The existence and uniqueness of solutions involving the delta shock wave for the Riemann problem is proven by employing the self-similar viscosity vanishing approach.

1. Introduction. In this paper, we are concerned with the Riemann problem for

$$
\left\{\begin{array}{l}
\partial_{t} v+\partial_{x}\left(\frac{v}{1+v}\right)=0 \\
\partial_{t} w+\partial_{x}\left(\frac{w}{1+v}\right)=0
\end{array}\right.
$$

with initial data

$$
(v, w)(x, 0)=\left(v_{ \pm}, w_{ \pm}\right), \quad \pm x>0
$$

where $v_{ \pm}$and $w_{ \pm}$are all given constants.

The equations (1.1) can be derived directly from the chromatography equations

$$
\left\{\begin{array}{l}
\partial_{t} u_{1}+\partial_{x}\left(\frac{u_{1}}{1+u_{1}+u_{2}}\right)=0 \\
\partial_{t} u_{2}+\partial_{x}\left(\frac{u_{2}}{1+u_{1}+u_{2}}\right)=0
\end{array}\right.
$$

by introducing the change of variables

$$
v=u_{1}+u_{2}, \quad w=u_{1}-u_{2} .
$$

Received October 8, 2009.

2000 Mathematics Subject Classification. Primary 35L65, 35L67, 35B30.

Key words and phrases. Delta shock wave; Riemann problem; viscosity method; Temple class; chromatography system; hyperbolic conservation laws.

This work is partially supported by the National Natural Science Foundation of China (10901077), the China Postdoctoral Science Foundation $(201003504,20090451089)$ and the Shandong Provincial Doctoral Foundation (BS2010SF006).

E-mail address: smnwhy0350@163.com 
Here $u_{1}, u_{2}$ are the nonnegative functions of the variables $(x, t) \in R \times R_{+}$, which express transformations of the concentrations of two solutes.

The system (1.3) belongs to the Temple class and arises in the study of two-component chromatography; i.e., the shock curves coincide with the rarefaction curves in the phase plane due to the special form $Y_{t}+(u Y)_{x}=0$ [29], where $Y=\left(u_{1}, u_{2}\right)^{T}$ and $u=$ $\frac{1}{1+u_{1}+u_{2}}$. Thanks to the above feature, well-posed results for Temple systems are available for a much larger class of initial data compared with general systems of conservation laws. Furthermore, the Riemann problem for these equations can be explicitly solved in the large, and wave interactions have a simplified structure. For the related results about Temple systems, we can refer to [3, 27] and the references cited therein.

Recently, Ambrosio et al. 2] introduced the change of variables (1.4) and then studied the equations (1.1) as an example by employing new well-posedness results for continuity and transport equations. Their method is mainly to split the chromatography system in the coupling between a scalar conservation law and a transport equation, and then the transport equation techniques [1] can be heavily exploited. A different change of variables $v=u_{1}+u_{2}$ and $\theta=u_{2} / u_{1}$ was adopted by Bressan and Shen [5], where they are mainly concerned with the study of ODEs with discontinuous vector fields.

In the present paper, we only consider the Riemann problem (1.1) and (1.2) and make a further step to assume that the functions $(v, w) \in R_{+} \times R$ from the mathematical viewpoint. We discover that no classical weak solutions appear when the Riemann initial data satisfy $v_{-}=0$ and the delta shock waves should be used as parts in the Riemann solutions. The motivation of this study comes from the fact that the delta shock wave was captured numerically and experimentally by Mazzotti et al. [20, 21, 22] in the Riemann solutions for the local equilibrium model of two-component nonlinear chromatography as follows:

$$
\left\{\begin{array}{c}
\partial_{t}\left(u_{1}+\frac{a_{1} u_{1}}{1-u_{1}+u_{2}}\right)+\partial_{x} u_{1}=0, \\
\partial_{t}\left(u_{2}+\frac{a_{2} u_{2}}{1-u_{1}+u_{2}}\right)+\partial_{x} u_{2}=0,
\end{array}\right.
$$

with certain Riemann initial data, in which $u_{1}$ and $u_{2}$ are the concentrations of the two absorbing species, and $u_{1}, u_{2} \geq 0,1-u_{1}+u_{2}>0, a_{2}>a_{1}>0$. This paper is a theoretical foundation for making a further step to study the Riemann problem of (1.5) in the future.

Furthermore, we show rigorously that the Riemann solution including the delta shock wave is the weak star limit of $\left(v^{\varepsilon}, w^{\varepsilon}\right)$ as $\varepsilon \rightarrow 0^{+}$, where $\left(v^{\varepsilon}, w^{\varepsilon}\right)$ is the self-similar solution for the corresponding Riemann problem of the perturbed system

$$
\left\{\begin{array}{l}
\partial_{t} v+\partial_{x}\left(\frac{v}{1+v}\right)=\varepsilon t \partial_{x x} v \\
\partial_{t} w+\partial_{x}\left(\frac{w}{1+v}\right)=0
\end{array}\right.
$$

Here our viscosity appears only in the first equation in (1.6) and our main interest is the weak convergence of the viscosity solutions for the delta shock wave. Thus the process of proof is given in detail for the case $v_{-}=0$ in which the delta shock wave occurs in the Riemann solution of (1.1) and (1.2). We can see that the structure of the delta shock wave is stable under the self-similar viscosity perturbation, which guarantees the delta shock wave be a unique entropy solution. 
The approach through self-similar viscosity limits was proposed by Dafermos [7] and used in Dafermos and Diperna [9] to construct solutions for broad classes of $2 \times 2$ systems that include the equations of isentropic, Lagrangian, gas dynamics. This method was widely used in [26, 30, for the admissibility of weak solutions of the Riemann problem for hyperbolic systems of conservation laws and the admissibility criterion was called the wave-fan criterion in [8]. Special attention was also paid in [12, 14, 25, 28, 31] to the formation of the delta shock waves in the Riemann solutions for some systems of hyperbolic conservation laws.

For knowledge of the delta shock wave, which was introduced into the classical weak solution by Korchinski when he studied the Riemann problem for

$$
\left\{\begin{array}{c}
u_{t}+\left(\frac{1}{2} u^{2}\right)_{x}=0, \\
v_{t}+\left(\frac{1}{2} u v\right)_{x}=0,
\end{array}\right.
$$

see [17]. In fact, the concept of the $\delta$-shock solution and the corresponding RankineHugoniot condition were also presented by Zeldovich and Myshkis [32] in the case of the continuity equation earlier. Tan, Zhang and Zheng [28] considered the reduced system, which has the trivial difference $u \rightarrow \frac{u}{2}$ with (1.7), and discovered that the form of the Dirac delta functions supported on shocks was used as parts in their Riemann solutions for certain initial data. Another well-known example is the transport equations (also called the pressureless Euler equations)

$$
\left\{\begin{array}{l}
\rho_{t}+(\rho u)_{x}=0, \\
(\rho u)_{t}+\left(\rho u^{2}\right)_{x}=0,
\end{array}\right.
$$

in which the delta shocks also appear in their Riemann solutions for certain initial data. The equations (1.8) have been studied extensively since 1994; for examples, we can see [4, 6, 11, 13, 15, 18, 19] and the related references therein.

The paper is organized in the following way. In section 2, the Riemann problem of (1.1) and (1.2) is constructed completely by employing the characteristic analysis method. In particular, the delta shock wave does occur in the Riemann solution when one of the Riemann initial data takes the special form $v_{-}=0$. In section 3 , the generalized RankineHugoniot relation of the delta shock wave is derived in detail, which describes the exact relationship among the location, propagation speed, weight and the assignment of $v$ on its discontinuity. In section 4, we consider the existence of the Riemann solutions to the viscous system (1.6) for each fixed $\varepsilon>0$ in the special case $v_{+}>v_{-}=0$. In addition, the solutions have the special form that $v^{\varepsilon}(\xi)$ is smooth and monotone increasing and that $w^{\varepsilon}(\xi)$ is smooth and monotone except for a unique singular point which is the unique fixed point of $\xi=\frac{1}{1+v^{\varepsilon}(\xi)}$. Finally we study the limit of the Riemann solution to (1.6) by letting $\varepsilon \rightarrow 0$ and prove that it is exactly the corresponding Riemann solution to (1.1) in the special case $v_{+}>v_{-}=0$ in section 5 . 
2. The Riemann problem for (1.1) and (1.2). In this section, we discuss the Riemann problem for (1.1) and (1.2). The characteristic roots of the equations (1.1) are

$$
\lambda_{1}=\frac{1}{(1+v)^{2}}, \quad \lambda_{2}=\frac{1}{1+v} .
$$

Therefore (1.1) is strictly hyperbolic in the half-plane of the phase space $v>0$ and nonstrictly hyperbolic on the borderline $v=0$.

The corresponding right characteristic vector of $\lambda_{i}(i=1,2)$ is

$$
\overrightarrow{r_{1}}=(v, w)^{T}, \quad \overrightarrow{r_{2}}=(0,1)^{T} .
$$

Obviously, we have $\nabla \lambda_{1} \cdot \overrightarrow{r_{1}} \neq 0$ for $v \neq 0$ and $\nabla \lambda_{2} \cdot \overrightarrow{r_{2}} \equiv 0$, which implies that $\lambda_{1}$ is genuinely nonlinear for $v \neq 0$ and $\lambda_{2}$ is always linearly degenerate. Therefore, the associated waves are rarefaction waves or shock waves for the first family and contact discontinuities for the second family.

The Riemann invariants along the characteristic fields are

$$
r=\frac{w}{v}, \quad s=v .
$$

Since both (1.1) and (1.2) are invariant under the transformation $(x, t) \rightarrow(\alpha x, \alpha t)$ ( $\alpha$ is constant), the solution of the Riemann problem must be a function of the single variable $x / t$. Thus, we seek the self-similar solution

$$
(v, w)(x, t)=(v, w)(\xi), \quad \xi=x / t .
$$

Then the Riemann problem of (1.1) and (1.2) is reduced to the boundary value problem of the ordinary differential equations:

$$
\left\{\begin{array}{l}
-\xi v_{\xi}+\left(\frac{v}{1+v}\right)_{\xi}=0, \\
-\xi w_{\xi}+\left(\frac{w}{1+v}\right)_{\xi}=0,
\end{array}\right.
$$

with $(v, w)( \pm \infty)=\left(v_{ \pm}, w_{ \pm}\right)$.

For smooth solutions, let us denote $U=(v, w)^{T}$. Then (2.5) can be rewritten as

$$
A(U) U_{\xi}=0,
$$

where

$$
A(v, w)=\left(\begin{array}{cc}
-\xi+\frac{1}{(1+v)^{2}} & 0 \\
-\frac{w}{(1+v)^{2}} & -\xi+\frac{1}{1+v}
\end{array}\right) .
$$

Besides the constant state solution, it provides a rarefaction wave which is a continuous solution of $(1.1)$ in the form $(v, w)(\xi)$. Then, for a given left state $\left(v_{-}, w_{-}\right)$, the rarefaction wave curves in the phase plane, which are the sets of states that can be connected on the right by a 1-rarefaction wave, are as follows:

$$
R\left(v_{-}, w_{-}\right):\left\{\begin{array}{l}
\xi=\lambda_{1}=\frac{1}{(1+v)^{2}}, \\
\frac{w}{v}=\frac{w_{-}}{v_{-}}, \quad v<v_{-} .
\end{array}\right.
$$


For a bounded discontinuity at $\xi=\sigma$, the Rankine-Hugoniot condition holds:

$$
\left\{\begin{array}{l}
-\sigma[v]+\left[\frac{v}{1+v}\right]=0, \\
-\sigma[w]+\left[\frac{w}{1+v}\right]=0,
\end{array}\right.
$$

where here and below, we use the usual notation $[v]=v_{r}-v_{l}$ with $v_{l}$ and $v_{r}$ the values of the function $v$ on the left-hand and right-hand sides of the discontinuity, etc.

If $v_{r} \neq v_{l}$, we have $\sigma_{1}=\frac{1}{\left(1+v_{r}\right)\left(1+v_{l}\right)}$ from the first equation in (2.8) and the Lax entropy conditions imply that $v_{l}<v_{r}$. Furthermore, we have the relation $\frac{w_{r}}{v_{r}}=\frac{w_{l}}{v_{l}}$ from the second equation in (2.8).

So for a given left state $\left(v_{-}, w_{-}\right)$, the sets of states which can be connected to $\left(v_{-}, w_{-}\right)$ by a 1 -shock wave on the right are as follows:

$$
S\left(v_{-}, w_{-}\right):\left\{\begin{array}{l}
\sigma_{1}=\frac{1}{(1+v)\left(1+v_{-}\right)}, \\
\frac{w}{v}=\frac{w_{-}}{v_{-}}, \quad v>v_{-} .
\end{array}\right.
$$

It is noted that the shock curves coincide with the rarefaction curves in the phase plane for (1.1) belongs to the Temple class.

If $v_{r}=v_{l}$, we can conclude that $\sigma_{2}=\frac{1}{1+v_{l}}=\frac{1}{1+v_{r}}$, which exactly corresponds to a contact discontinuity of the second family. Since $\lambda_{2}$ is linearly degenerate, the sets of states can be connected to a given left state $\left(v_{-}, w_{-}\right)$by a contact discontinuity $J$ on the right if and only if $v=v_{-}$.

Thus, we can summarize that the sets of states connected on the right consist of the 1rarefaction wave curve $R\left(v_{-}, w_{-}\right)$, the 1 -shock wave curve $S\left(v_{-}, w_{-}\right)$and the 2-contact discontinuity $J\left(v_{-}, w_{-}\right)$for a given left state $\left(v_{-}, w_{-}\right)$. These curves divide the half phase plane $v>0$ into two regions $\mathrm{I}=\left\{(v, w) \mid 0<v<v_{-}\right\}$and $\mathrm{I}=\left\{(v, w) \mid v>v_{-}\right\}$(see Fig. 1). According to the right state $\left(v_{+}, w_{+}\right)$in the different region, one can construct the unique global Riemann solution connecting two constant states $\left(v_{ \pm}, w_{ \pm}\right)$.
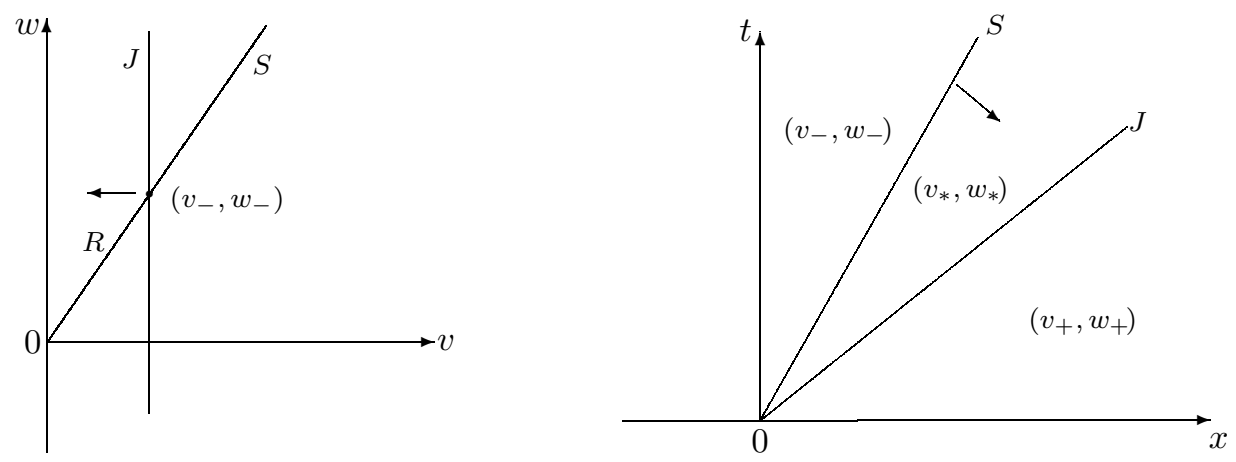

FIG. 1

Clearly the Riemann solution is $R+J$ if $v_{+}<v_{-}$; otherwise the Riemann solution should be $S+J$ if $v_{+}>v_{-}>0$. For the case $v_{+}>v_{-}=0$, we should consider the limit of solutions $(v, w)(\xi)$ when $v_{+}, w_{-}, w_{+}$are fixed, $v_{+}>0$ and $v_{-} \rightarrow 0+$. When 
$v_{+}>v_{-}>0$, the solution is shown in Fig. 1 and the intermediate state can be calculated by $\left(v_{*}, w_{*}\right)=\left(v_{+}, \frac{w_{-} v_{+}}{v_{-}}\right)$. When $v_{-} \rightarrow 0+$, it is obvious to see that $w_{*}=\frac{w_{-} v_{+}}{v_{-}} \rightarrow \infty$. At the same time, the speed of the shock wave $\sigma_{1}=\frac{1}{\left(1+v_{+}\right)\left(1+v_{-}\right)}$tends to that of the contact discontinuity $\sigma_{2}=\frac{1}{1+v_{+}}$as $v_{-} \rightarrow 0+$, which means that $S$ and $J$ coincide to form a new type of nonlinear hyperbolic wave, which is called the delta shock wave in 28 .

Now, let us calculate the total quantity of $w$ between $S$ and $J$ as $v_{-} \rightarrow 0+$ as follows:

$$
\lim _{v_{-} \rightarrow 0} \int_{\sigma_{1}-0}^{\sigma_{2}+0} w_{*} d \xi=\lim _{v_{-} \rightarrow 0+} \int_{\frac{1}{\left(1+v_{+}\right)\left(1+v_{-}\right)}}^{\frac{1}{\left(1+v_{+}\right)}} \frac{w_{-} v_{+}}{v_{-}} d \xi .
$$

Integrating the second equation in $(2.5)$ with respect to $\xi$ from $\sigma_{1}-0$ to $\sigma_{2}+0$, we have

$$
\begin{aligned}
0 & =\int_{\sigma_{1}-0}^{\sigma_{2}+0}-\xi d w+d \frac{w}{1+v} \\
& =-\left.\xi w\right|_{\sigma_{1}-0} ^{\sigma_{2}+0}+\int_{\sigma_{1}-0}^{\sigma_{2}+0} w d \xi+\left.\frac{w}{1+v}\right|_{\sigma_{1}-0} ^{\sigma_{2}+0}
\end{aligned}
$$

An easy computation leads to

$$
\lim _{v_{-} \rightarrow 0} \int_{\sigma_{1}-0}^{\sigma_{2}+0} w_{*} d \xi=\lim _{v_{-} \rightarrow 0+} \frac{w_{-} v_{+}}{\left(1+v_{-}\right)\left(1+v_{+}\right)}=\frac{w_{-} v_{+}}{1+v_{+}},
$$

which means that $w(\xi)$ has the same singularity as a weighted Dirac delta function at $\xi=\frac{1}{1+v_{+}}$when $v_{-}=0$. Motivated by [28], we should seek the Riemann solutions of (1.1) and (1.2) including the delta shock wave in the special case $v_{-}=0$.

3. The generalized Rankine-Hugoniot relations of the delta shock wave. In the case $v_{-}=0$, from [19, 25, 28, we know that the singularity is impossible to be a jump with finite amplitude, which implies that there is no solution which is piecewise smooth and bounded. Hence the Riemann solution containing a weighted $\delta$-measure supported on a line should be constructed.

In order to define the measure solutions, the two-dimensional weighted $\delta$-measure $\beta(s) \delta_{\Gamma}$ supported on a smooth curve $\Gamma=\{(x(s), t(s)): a<s<b\}$ can be defined by

$$
\left\langle\beta(s) \delta_{\Gamma}, \psi(x, t)\right\rangle=\int_{a}^{b} \beta(s) \psi(x(s), t(s)) d s,
$$

for any test function $\psi(x, t) \in C_{0}^{\infty}\left(R \times R_{+}\right)$.

With the above definition, a family of $\delta$-measure solutions $(v, w)$ of (1.1) and (1.2) with the parameter $\sigma$ in the case $v_{+}>v_{-}=0$ can be expressed as

$$
v(x, t)=v_{+} H(x-\sigma t), \quad w(x, t)=w_{-}+[w] H(x-\sigma t)+\beta(t) \delta_{\Gamma},
$$

in which $\Gamma=\{(\sigma t, t): 0 \leq t<+\infty\}, \beta(t)=\left(\sigma[w]-\left[\frac{w}{1+v}\right]\right) t$ and $H(x)$ is the Heaviside function. 
According to [6, 25], the $\delta$-measure solutions $(v, w)$ constructed above should satisfy

$$
\left\{\begin{array}{l}
\left\langle v, \psi_{t}\right\rangle+\left\langle\frac{v}{1+v}, \psi_{x}\right\rangle=0 \\
\left\langle w, \psi_{t}\right\rangle+\left\langle\frac{w}{1+v}, \psi_{x}\right\rangle=0
\end{array}\right.
$$

for all test functions $\psi(x, t) \in C_{0}^{\infty}\left(R \times R_{+}\right)$, in which

$$
\begin{aligned}
& \langle w, \psi\rangle=\int_{0}^{\infty} \int_{-\infty}^{\infty} w_{0} \psi d x d t+\left\langle\beta(t) \delta_{\Gamma}, \psi\right\rangle, \\
& \left\langle\frac{w}{1+v}, \psi\right\rangle=\int_{0}^{\infty} \int_{-\infty}^{\infty} \frac{w_{0} \psi}{1+v_{0}} d x d t+\left\langle\sigma \beta(t) \delta_{\Gamma}, \psi\right\rangle .
\end{aligned}
$$

Here $v_{0}=v_{+} H(x-\sigma t)$ and $\frac{w_{0}}{1+v_{0}}=w_{-}+\left[\frac{w}{1+v}\right] H(x-\sigma t)$.

Theorem 3.1. For the case $v_{+}>v_{-}=0$, the Riemann solution of (1.1) and (1.2) is piecewise smooth in the form

$$
(v, w)(x, t)= \begin{cases}\left(0, w_{-}\right), & x<\sigma t \\ \left(v_{\delta}, \beta(t) \delta(x-\sigma t)\right), & x=\sigma t \\ \left(v_{+}, w_{+}\right), & x>\sigma t\end{cases}
$$

where

$$
v_{\delta}=v_{+}, \quad \sigma=\frac{1}{1+v_{+}}, \quad \beta(t)=\frac{w_{-} v_{+} t}{1+v_{+}} .
$$

The measure solution (3.6) together with (3.7) also satisfies the generalized RankineHugoniot condition as follows:

$$
\left\{\begin{array}{l}
\frac{d x}{d t}=\sigma \\
\frac{d \beta(t)}{d t}=[w] \sigma-\left[\frac{w}{1+v}\right], \\
{[v] \sigma=\left[\frac{v}{1+v}\right]}
\end{array}\right.
$$

and

$$
\sigma=\frac{1}{1+v_{\delta}} .
$$

Proof. At first we should check that the above constructed $\delta$-measure solution (3.6) with (3.7) should satisfy the equations (1.1) in the sense of distributions. Based on the definition of the Schwarz distribution, this is equivalent to proving that (3.6) and (3.7) should satisfy

$$
\left\{\begin{array}{l}
\int_{0}^{\infty} \int_{-\infty}^{\infty}\left(v \psi_{t}+\frac{v}{1+v} \psi_{x}\right) d x d t=0 \\
\int_{0}^{\infty} \int_{-\infty}^{\infty}\left(w \psi_{t}+\frac{w}{1+v} \psi_{x}\right) d x d t=0
\end{array}\right.
$$


Actually, we have

$$
\begin{aligned}
& \int_{0}^{\infty} \int_{-\infty}^{\infty}\left(v \psi_{t}+\frac{v}{1+v} \psi_{x}\right) d x d t \\
& =\int_{0}^{\infty} \int_{-\infty}^{x(t)}\left(v_{-} \psi_{t}+\frac{v_{-}}{1+v_{-}} \psi_{x}\right) d x d t+\int_{0}^{\infty} \int_{x(t)}^{\infty}\left(v_{+} \psi_{t}+\frac{v_{+}}{1+v_{+}} \psi_{x}\right) d x d t \\
& =\int_{0}^{\infty} \int_{0}^{t(x)} v_{+} \psi_{t} d t d x-\int_{0}^{\infty} \frac{v_{+}}{1+v_{+}} \psi\left(\frac{t}{1+v_{+}}, t\right) d t \\
& =\int_{0}^{\infty} v_{+} \psi\left(x,\left(1+v_{+}\right) x\right) d x-\int_{0}^{\infty} \frac{v_{+}}{1+v_{+}} \psi\left(\frac{t}{1+v_{+}}, t\right) d t=0 .
\end{aligned}
$$

On the other hand, we can also calculate

$$
\begin{aligned}
\int_{0}^{\infty} \int_{-\infty}^{\infty}\left(w \psi_{t}+\frac{w}{1+v} \psi_{x}\right) d x d t \\
=\int_{0}^{\infty} \int_{-\infty}^{x(t)}\left(w_{-} \psi_{t}+\frac{w_{-}}{1+v_{-}} \psi_{x}\right) d x d t+\int_{0}^{\infty} \int_{x(t)}^{\infty}\left(w_{+} \psi_{t}+\frac{w_{+}}{1+v_{+}} \psi_{x}\right) d x d t \\
\quad+\int_{0}^{\infty} \beta(t)\left(\psi_{t}\left(\frac{t}{1+v_{+}}, t\right)+\frac{1}{1+v_{\delta}} \psi_{x}\left(\frac{t}{1+v_{+}}, t\right)\right) d t \\
=\int_{0}^{\infty}-w_{-} \psi\left(x,\left(1+v_{+}\right) x\right) d x+\int_{0}^{\infty} w_{-} \psi\left(\frac{t}{1+v_{+}}, t\right) d t+\int_{0}^{\infty} w_{+} \psi\left(x,\left(1+v_{+}\right) x\right) d x \\
-\int_{0}^{\infty} \frac{w_{+}}{1+v_{+}} \psi\left(\frac{t}{1+v_{+}}, t\right) d t+\int_{0}^{\infty} \beta(t) d \psi\left(\frac{t}{1+v_{+}}, t\right) \\
=\left(\frac{-w_{-}}{1+v_{+}}+w_{-}+\frac{w_{+}}{1+v_{+}}-\frac{w_{+}}{1+v_{+}}-\frac{w_{-} v_{+}}{1+v_{+}}\right) \int_{0}^{\infty} \psi\left(\frac{t}{1+v_{+}}, t\right) d t=0 .
\end{aligned}
$$

From the above, we can see that (3.6) and (3.7) is indeed the piecewise smooth Riemann solution of (1.1) and (1.2) in the sense of distributions. It is clear to see that the generalized Rankine-Hugoniot conditions (3.8) with (3.9) hold by substituting (3.6) and (3.7) into (3.8) and (3.9) to clarify.

The left-hand side of the second equation in (3.8) is called the Rankine-Hugoniot deficit in [16]. The second integral equality in (3.10) differs from the classical situation by the additional term $\int_{0}^{\infty} \beta(t) d \psi\left(\frac{t}{1+v_{+}}, t\right)$ due to the Rankine-Hugoniot deficit. The relation (3.8) together with (3.9) is called the generalized Rankine-Hugoniot relation of the delta shock wave, which reflects the exact relationship among the location, propagation speed, weight and the assignment of $v$ on its discontinuity.

In order to ensure the uniqueness, the $\delta$-entropy condition

$$
\lambda_{1+}<\lambda_{2+}=\sigma<\lambda_{1-}=\lambda_{2-}
$$

should be proposed, which means that none of the four characteristic lines on both sides of the $\delta$-shock wave curve is outgoing here. More precisely, the entropy condition for 
the $\delta$-shock wave here is that three characteristic lines enter the discontinuity while the remaining one is tangent to the discontinuity.

Now, we employ the generalized Rankine-Hugoniot relations (3.8) and (3.9) to solve the Riemann problem of (1.1) and (1.2) with $v_{-}=0$ and the initial data become $t=$ $0: x(0)=0, \beta(0)=0, v_{\delta}(0)=0$. In view of our knowledge about delta shock waves, we know that $\sigma, v_{\delta}$ are constants and $\beta(t)$ is a linear function of $t$. Under the $\delta$-entropy condition (3.11), it is easy to check that $\sigma, v_{\delta}$ and $\beta(t)$ can be determined uniquely.

Motivated by [10, 23, 24, we simply consider here the geometrical and physical sense of the generalized Rankine-Hugoniot condition of the delta shock wave for (1.1). It is well known that if a pair of compactly supported functions $v(x, t), w(x, t) \in L^{\infty}\left(R \times R_{+}\right)$ with respective to $x$ is a generalized solution of (1.1), then we have

$$
\int_{-\infty}^{+\infty} v(x, t) d x=\int_{-\infty}^{+\infty} v_{0}(x) d x, \quad \int_{-\infty}^{+\infty} w(x, t) d x=\int_{-\infty}^{+\infty} w_{0}(x) d x
$$

which are independent of time and here $\left(v_{0}(x), w_{0}(x)\right)$ is the initial state.

However this fact does not hold for a $\delta$-shock wave type solution. Suppose that $(v(x, t), w(x, t))$ is a generalized $\delta$-shock wave type solution of the Cauchy problem for (1.1) with $\delta$-shock wave type initial data. Here $w(x, t)=W(x, t)+\beta(t) \delta(\Gamma)$, $\Gamma:\{(x, t) \mid x=\phi(t)\}$ is the discontinuity and $v(x, t), W(x, t)$ are compactly supported functions with respect to $x$. Let us denote

$$
S_{v}(t)=\int_{-\infty}^{\phi(t)} v(x, t) d x+\int_{\phi(t)}^{+\infty} v(x, t) d x, \quad S_{w}(t)=\int_{-\infty}^{\phi(t)} w(x, t) d x+\int_{\phi(t)}^{+\infty} w(x, t) d x .
$$

Then we have the following balance relations:

$$
\dot{S}_{v}(t)=0, \quad \dot{S}_{w}(t)=-\dot{\beta}(t)=-[w] \sigma+\left[\frac{w}{1+v}\right] .
$$

It is clear from (3.14) that the sense of strength $\beta(t)$ of the delta shock wave is the "area" of the discontinuity line and the "total area" $S_{w}(t)+\beta(t)$ is independent of time $t$.

4. Existence of solutions for the viscous system (1.6). In this section, we mainly consider the existence of the Riemann solutions of (1.6) and (1.2). For convenience and conciseness, we only consider the case $v_{+}>v_{-}=0$. By performing the self-similar transformation $\xi=x / t$, it can be transferred into the boundary value problem:

$$
\left\{\begin{array}{l}
-\xi v_{\xi}+\left(\frac{v}{1+v}\right)_{\xi}=\varepsilon v_{\xi \xi} \\
-\xi w_{\xi}+\left(\frac{w}{1+v}\right)_{\xi}=0
\end{array}\right.
$$

with the boundary conditions

$$
(v, w)( \pm \infty)=\left(v_{ \pm}, w_{ \pm}\right) .
$$

Since the first equation in (4.1) does not depend on the solution of the second one, let us first consider the first equation in (4.1), i.e., let us solve

$$
\left\{\begin{array}{l}
-\xi v_{\xi}+\left(\frac{v}{1+v}\right)_{\xi}=\varepsilon v_{\xi \xi} \\
v( \pm \infty)=v_{ \pm}
\end{array}\right.
$$


Now we can show the existence, uniqueness and monotonicity for the smooth solution of (4.3) in the following theorem, which is a special version of the case $m=1$ in [7].

Theorem 4.1. For each fixed $\varepsilon>0$, there exists a unique and monotonic smooth solution of the boundary value problem (4.3) for $v_{+}>v_{-}=0$.

Proof. In order to investigate the monotonicity and existence, we adopt the Dafermos technique and start with the following altered problem:

$$
\left\{\begin{array}{l}
-\xi v_{\xi}+\mu\left(\frac{v}{1+v}\right)_{\xi}=\varepsilon v_{\xi \xi} \\
v( \pm R)=\mu v_{ \pm}
\end{array}\right.
$$

where $\mu \in[0,1]$ and $R$ is a sufficiently large real number.

Integrating (4.4) with respect to $\xi$ from $\xi_{0}$ to $\xi$, where $\left[\xi_{0}, \xi\right] \subseteq[-R, R]$, we have

$$
\dot{v}^{\varepsilon}(\xi)=\dot{v}^{\varepsilon}\left(\xi_{0}\right) \exp \left(\frac{1}{\varepsilon} \int_{\xi_{0}}^{\xi}\left[\frac{\mu}{(1+v(s))^{2}}-s\right] d s\right) .
$$

From (4.5), it is clear that $\dot{v}^{\varepsilon}(\xi) / \dot{v}^{\varepsilon}\left(\xi_{0}\right)>0$ for arbitrary $\left[\xi_{0}, \xi\right] \subseteq[-R, R]$. Thus the solution of (4.4) is monotonically increasing in the interval $[-R, R]$ based on $v_{+}>v_{-}=0$.

Now we consider the existence of the solution of (4.4). Since the solution of (4.4) is monotonically increasing in the interval $[-R, R]$, we have

$$
\sup _{\xi \in[-R, R]}\left\|v^{\varepsilon}(\xi)\right\|_{L^{\infty}}=\sup _{\xi \in[-R, R]}\left|v^{\varepsilon}(\xi)\right| \leq v_{+},
$$

which is obviously independent of $\mu$ and $R$. From Theorem 3.1 in [7, we know that there exists a solution of (4.3) which is monotonically increasing in $(-\infty,+\infty)$.

Eventually, we consider the uniqueness of the solutions of (4.3) and assume that $v_{1}^{\varepsilon}(\xi)$ and $v_{2}^{\varepsilon}(\xi)$ are two smooth solutions of (4.3). Let us denote $h(\xi)=v_{2}^{\varepsilon}(\xi)-v_{1}^{\varepsilon}(\xi)$, which should satisfy the following boundary value problem:

$$
\left\{\begin{array}{l}
-\xi h_{\xi}+(h g)_{\xi}=\varepsilon h_{\xi \xi}, \\
h( \pm \infty)=0 .
\end{array}\right.
$$

Here we denote $f(v)=\frac{v}{1+v}$ and $g(\xi)$ can be calculated by

$$
g(\xi)=\frac{f\left(v_{2}^{\varepsilon}(\xi)\right)-f\left(v_{1}^{\varepsilon}(\xi)\right)}{v_{2}^{\varepsilon}(\xi)-v_{1}^{\varepsilon}(\xi)}=\frac{1}{\left(1+v_{1}^{\varepsilon}(\xi)\right)\left(1+v_{2}^{\varepsilon}(\xi)\right)} .
$$

Let us suppose that $h(\xi)$ is not the null function and let $\alpha$ and $\beta$ be the two consecutive zero points of $h(\xi)$ with $-\infty \leq \alpha<\beta \leq+\infty$. In order to integrate (4.7) by parts, we should prove that $\lim _{\xi \rightarrow \alpha-} \xi h(\xi)=0$ (or/and $\lim _{\xi \rightarrow \beta+} \xi h(\xi)=0$ ) if $\alpha=-\infty$ (or/and $\beta=+\infty$ ).

Multiplying the first equation in (4.1) by $\exp \frac{\xi^{2}}{2 \varepsilon}$, we have

$$
\frac{d}{d \xi}\left(\dot{v} \exp \frac{\xi^{2}}{2 \varepsilon}\right)=\frac{1}{(1+v)^{2}}\left(\dot{v} \exp \frac{\xi^{2}}{2 \varepsilon}\right) .
$$

Keeping $0 \leq v^{\varepsilon}(\xi) \leq v_{+}$and $\dot{v}(\xi) \geq 0$ in mind, it follows that

$$
\dot{v}^{\varepsilon}(\xi) \leq \dot{v}^{\varepsilon}(0) \exp \left(\frac{2|\xi|-\xi^{2}}{2 \varepsilon}\right)
$$


Since both $v_{1}^{\varepsilon}(\xi)$ and $v_{2}^{\varepsilon}(\xi)$ are smooth solutions of (4.3), we have

$$
|\dot{h}(\xi)|=\left|\dot{v}_{2}^{\varepsilon}(\xi)-\dot{v}_{1}^{\varepsilon}(\xi)\right| \leq\left(\dot{v}_{1}^{\varepsilon}(0)+\dot{v}_{2}^{\varepsilon}(0)\right) \exp \left(\frac{2|\xi|-\xi^{2}}{2 \varepsilon}\right),
$$

which implies that $\dot{h}(\xi)$ decays rapidly to zero when $|\xi| \rightarrow \infty$ for each fixed $\varepsilon>0$. Thus we have $\lim _{\xi \rightarrow \pm \infty} \xi h(\xi)=0$ if $\lim _{\xi \rightarrow \pm \infty} h(\xi)=0$.

Noting that $g(\xi)$ is bounded, integrating (4.7) by parts in the interval $(\alpha, \beta)$, we can obtain

$$
\int_{\alpha}^{\beta} h(\xi) d \xi=\varepsilon(\dot{h}(\beta)-\dot{h}(\alpha)) .
$$

If $h(\xi)>0$ in $(\alpha, \beta)$, it is easy to get $\dot{h}(\beta) \leq 0 \leq \dot{h}(\alpha)$ and $\int_{\alpha}^{\beta} h(\xi) d \xi>0$, which obviously contradicts (4.12). We have the same contradiction if $h(\xi)<0$ in $(\alpha, \beta)$; thus we arrive at $h(\xi)=0$.

Now, we consider the existence of the solutions $w(\xi)$ for the second equation in (4.1) based on Theorem 4.1 and have the following theorem.

Theorem 4.2. For each fixed $\varepsilon>0$, assume that $w(\xi)$ is a weak solution of the following boundary value problem:

$$
\left\{\begin{array}{l}
-\xi w_{\xi}+\left(\frac{w}{1+v^{\varepsilon}}\right)_{\xi}=0 \\
w( \pm \infty)=w_{ \pm}
\end{array}\right.
$$

where $v^{\varepsilon}(\xi)$ is the solution of (4.3).

Then, we have $w^{\varepsilon}(\xi) \in L^{1}(-\infty,+\infty)$, which can be expressed as

$$
w^{\varepsilon}(\xi)= \begin{cases}w_{-} \exp \left(\int_{-\infty}^{\xi} \frac{\dot{v}^{\varepsilon}(s)}{1+v^{\varepsilon}(s)-s\left(1+v^{\varepsilon}(s)\right)^{2}}\right) d s, & \text { for } \xi<\xi_{\alpha}^{\varepsilon}, \\ w_{+} \exp \left(\int_{\xi}^{\infty} \frac{-\dot{v}^{\varepsilon}(s)}{1+v^{\varepsilon}(s)-s\left(1+v^{\varepsilon}(s)\right)^{2}}\right) d s, & \text { for } \xi>\xi_{\alpha}^{\varepsilon},\end{cases}
$$

where $\xi_{\alpha}^{\varepsilon}$ is the unique fixed point of $\xi=\frac{1}{1+v^{\varepsilon}(\xi)}$.

Proof. Since $v^{\varepsilon}(\xi)$ is monotonically increasing, the singularity point of (4.13) is unique and can be given by the solution of $\xi=\frac{1}{1+v^{\varepsilon}(\xi)}$; here we denote it with $\xi_{\alpha}^{\varepsilon}$. The formula (4.14) can be obtained through integrating the equation (4.13) from $-\infty$ to $\xi$ for $\xi<\xi_{\alpha}^{\varepsilon}$ and from $\xi$ to $\infty$ for $\xi>\xi_{\alpha}^{\varepsilon}$, respectively. From (4.14), it is easy to see that $w^{\varepsilon}(\xi)$ is monotone increasing $\left(w_{-}>0\right)$ or monotone decreasing $\left(w_{-}<0\right)$ in the interval $\left(-\infty, \xi_{\alpha}^{\varepsilon}\right)$, and is monotone decreasing $\left(w_{+}>0\right)$ or monotone increasing $\left(w_{+}<0\right)$ in the interval $\left(\xi_{\alpha}^{\varepsilon},+\infty\right)$. Furthermore, we have

$$
\begin{array}{ll}
\lim _{\xi \rightarrow \xi_{\alpha}^{\varepsilon}-} w^{\varepsilon}(\xi)= \pm \infty & \left(w_{-} \gtrless 0\right), \\
\lim _{\xi \rightarrow \xi_{\alpha}^{\varepsilon}+} w^{\varepsilon}(\xi)= \pm \infty & \left(w_{+} \gtrless 0\right) .
\end{array}
$$

Thus, the solution of (4.13) can be obtained by pasting together the two solutions in the intervals $\left(-\infty, \xi_{\alpha}^{\varepsilon}\right)$ and $\left(\xi_{\alpha}^{\varepsilon}, \infty\right)$. 
Now we proceed to prove that $w^{\varepsilon}(\xi)$ is a weak solution of (4.13). Integrating the equation (4.13) on $[-R, \xi]$ for $-R<\xi<\xi_{\alpha}^{\varepsilon}$, we have

$$
\int_{-R}^{\xi} w(s) d s+w(\xi)\left(\frac{1}{1+v^{\varepsilon}(\xi)}-\xi\right)=w(-R)\left(\frac{1}{1+v^{\varepsilon}(-R)}+R\right) .
$$

Let us denote

$$
p(\xi)=\int_{-R}^{\xi} w(s) d s, \quad a(\xi)=\frac{1}{1+v^{\varepsilon}(\xi)}-\xi, \quad A=w(-R)\left(\frac{1}{1+v^{\varepsilon}(-R)}+R\right) .
$$

Then, the equation (4.17) can be rewritten as

$$
\left\{\begin{array}{l}
a(\xi) p^{\prime}(\xi)+p(\xi)=A, \\
p(-R)=0,
\end{array}\right.
$$

and an easy computation leads to

$$
p(\xi)=A\left[1-\exp \left(-\int_{-R}^{\xi} \frac{d s}{a(s)}\right)\right] .
$$

Noting that $a(\xi)=O\left(\left|\xi-\xi_{\alpha}^{\varepsilon}\right|\right)$ as $\xi \rightarrow \xi_{\alpha}^{\varepsilon}-$, we have

$$
\lim _{\xi \rightarrow \xi_{\alpha}^{\varepsilon}-0} \int_{-R}^{\xi} \frac{d s}{a(s)}=+\infty
$$

Hence, passing to the limit $\xi \rightarrow \xi_{\alpha}^{\varepsilon}-$ in (4.19), it follows that

$$
\lim _{\xi \rightarrow \xi_{\alpha}^{\varepsilon}-0} \int_{-R}^{\xi} w(s) d s=\lim _{\xi \rightarrow \xi_{\alpha}^{\varepsilon}-0} p(\xi)=A .
$$

In view of (4.17), it is easy to see that

$$
\lim _{\xi \rightarrow \xi_{\alpha}^{\varepsilon}-0} w(\xi)\left(\frac{1}{1+v^{\varepsilon}(\xi)}-\xi\right)=0 .
$$

With the same reason as before, we also have

$$
\begin{gathered}
\lim _{\xi \rightarrow \xi_{\alpha}^{\varepsilon}+0} \int_{\xi}^{R} w(s) d s=w(R)\left(R-\frac{1}{1+v^{\varepsilon}(R)}\right), \\
\lim _{\xi \rightarrow \xi_{\alpha}^{\varepsilon}+0} w(\xi)\left(\xi-\frac{1}{1+v^{\varepsilon}(\xi)}\right)=0 .
\end{gathered}
$$

The equalities (4.21) and (4.23) mean that $w^{\varepsilon}(\xi) \in L^{1}[-R,+R]$.

Now, for an arbitrary test function $\psi \in C_{0}^{\infty}[-R, R]$, let us prove

$$
\left\langle-\xi w_{\xi}+\left(\frac{w}{1+v^{\varepsilon}}\right)_{\xi}, \psi\right\rangle=0 .
$$


Indeed, for arbitrary $\xi_{1}, \xi_{2}$ satisfying $-R<\xi_{1}<\xi_{\alpha}^{\varepsilon}<\xi_{2}<R$, we have

$$
\begin{aligned}
I & =\int_{-R}^{R}\left(w(\xi)\left(\xi-\frac{1}{1+v^{\varepsilon}(\xi)}\right) \psi^{\prime}(\xi)+w(\xi) \psi(\xi)\right) d \xi \\
& =\left\{\int_{-R}^{\xi_{1}}+\int_{\xi_{1}}^{\xi_{2}}+\int_{\xi_{2}}^{R}\right\}\left(w(\xi)\left(\xi-\frac{1}{1+v^{\varepsilon}(\xi)}\right) \psi^{\prime}(\xi)+w(\xi) \psi(\xi)\right) d \xi \\
& =I_{1}+I_{2}+I_{3} .
\end{aligned}
$$

Noting that

$$
-\left(w(\xi)\left(\xi-\frac{1}{1+v^{\varepsilon}(\xi)}\right)\right)_{\xi}+w(\xi)=-\xi w_{\xi}+\left(\frac{w}{1+v^{\varepsilon}}\right)_{\xi}=0,
$$

through integrating by parts, from (4.22) and (4.24), we can estimate as follows:

$$
\begin{aligned}
& \left|I_{1}\right|=\left|w\left(\xi_{1}\right)\left(\xi_{1}-\frac{1}{1+v^{\varepsilon}\left(\xi_{1}\right)}\right) \psi\left(\xi_{1}\right)\right| \rightarrow 0 \quad \text { as } \quad \xi_{1} \rightarrow \xi_{\alpha}^{\varepsilon}- \\
& \left|I_{3}\right|=\left|w\left(\xi_{2}\right)\left(\xi_{2}-\frac{1}{1+v^{\varepsilon}\left(\xi_{2}\right)}\right) \psi\left(\xi_{2}\right)\right| \rightarrow 0 \quad \text { as } \quad \xi_{2} \rightarrow \xi_{\alpha}^{\varepsilon}+.
\end{aligned}
$$

For $w(\xi) \in L^{1}[-R,+R]$, we have

$$
\left|I_{2}\right| \leq \int_{\xi_{1}}^{\xi_{2}}|w(\xi)| \cdot\left|\left(\xi-\frac{1}{1+v^{\varepsilon}(\xi)}\right) \psi^{\prime}(\xi)+\psi(\xi)\right| d \xi \rightarrow 0
$$

as $\xi_{1} \rightarrow \xi_{\alpha}^{\varepsilon}-$ and $\xi_{2} \rightarrow \xi_{\alpha}^{\varepsilon}+$.

Thus $I=0$ is independent of $\xi_{1}$ and $\xi_{2}$ in the sense of distributions; namely (4.25) is true. Since $R$ is arbitrarily a sufficiently large real number, $w^{\varepsilon}(\xi)$ defined in (4.14) is the unique weak solution of (4.13).

5. Convergence of the viscous solutions. Let us denote $\xi_{\alpha}$ to express the limit of the unique fixed point $\xi_{\alpha}^{\varepsilon}$ satisfying $\xi_{\alpha}^{\varepsilon}=\left(1+v^{\varepsilon}\left(\xi_{\alpha}^{\varepsilon}\right)\right)^{-1}$ in section 4 and let $\xi_{\beta}$ express the limit of the unique fixed point $\xi_{\beta}^{\varepsilon}$ satisfying $\xi_{\beta}^{\varepsilon}=\left(1+v^{\varepsilon}\left(\xi_{\beta}^{\varepsilon}\right)\right)^{-2}$ as $\varepsilon \rightarrow 0^{+}$. These points will play a very important role in the following discussion.

Lemma 5.1. For arbitrary $\eta>0$, we can conclude that

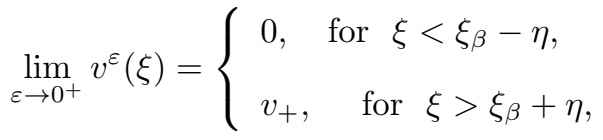

uniformly in the above intervals.

Proof. Let us take $\xi_{1}=\xi_{\beta}+\eta / 2$ and $\varepsilon>0$ sufficiently small so that $\xi_{\beta}^{\varepsilon}<\xi_{1}-\eta / 4$. Integrating (4.3) over $\left(\xi_{1}, \xi\right)$ yields

$$
\dot{v}^{\varepsilon}(\xi)=\dot{v}^{\varepsilon}\left(\xi_{1}\right) \exp \int_{\xi_{1}}^{\xi} \frac{1}{\varepsilon}\left(-s+\frac{1}{\left(1+v^{\varepsilon}(s)\right)^{2}}\right) d s .
$$


Integrating (5.2) over $\left(\xi_{1},+\infty\right)$ again, we have

$$
v_{+}-v^{\varepsilon}\left(\xi_{1}\right)=\dot{v}^{\varepsilon}\left(\xi_{1}\right) \int_{\xi_{1}}^{+\infty}\left(\exp \int_{\xi_{1}}^{\xi} \frac{1}{\varepsilon}\left(-s+\frac{1}{\left(1+v^{\varepsilon}(s)\right)^{2}}\right) d s\right) d \xi .
$$

Since $v^{\varepsilon}(\xi)$ is monotonically increasing in the real axis, we have $\dot{v}^{\varepsilon}\left(\xi_{1}\right) \geq 0$ and $0 \leq v^{\varepsilon}(s) \leq v_{+}$. Now we can estimate that

$$
\begin{aligned}
v_{+} & \geq \dot{v}^{\varepsilon}\left(\xi_{1}\right) \int_{\xi_{1}}^{+\infty}\left(\exp \int_{\xi_{1}}^{\xi} \frac{1}{\varepsilon}\left(-s+\frac{1}{\left(1+v_{+}\right)^{2}}\right) d s\right) d \xi \\
& =\dot{v}^{\varepsilon}\left(\xi_{1}\right) \int_{\xi_{1}}^{+\infty} \exp \frac{1}{\varepsilon}\left(-\frac{1}{2}\left(\xi-\xi_{1}\right)^{2}+\frac{\xi-\xi_{1}}{\left(1+v_{+}\right)^{2}}\right) d \xi \\
& =\dot{v}^{\varepsilon}\left(\xi_{1}\right) \int_{0}^{+\infty} \exp \frac{1}{\varepsilon}\left(-\frac{1}{2} \xi^{2}+\frac{\xi}{\left(1+v_{+}\right)^{2}}\right) d \xi \\
& \geq \dot{v}^{\varepsilon}\left(\xi_{1}\right) \cdot \varepsilon C
\end{aligned}
$$

for $0<\varepsilon<1$ and $C$ is a constant independent of $\varepsilon$. Thus we have $0 \leq \dot{v}^{\varepsilon}\left(\xi_{1}\right) \leq \frac{v_{+}}{\varepsilon C}$, and together with (5.2), we can estimate that

$$
\dot{v}^{\varepsilon}(\xi) \leq \frac{v_{+}}{\varepsilon C} \exp \int_{\xi_{1}}^{\xi} \frac{1}{\varepsilon}\left(-s+\frac{1}{\left(1+v^{\varepsilon}(s)\right)^{2}}\right) d s .
$$

Furthermore, we have

$$
-s+\frac{1}{\left(1+v^{\varepsilon}(s)\right)^{2}}=\frac{1}{\left(1+v^{\varepsilon}(s)\right)^{2}}-\frac{1}{\left(1+v^{\varepsilon}\left(\xi_{\beta}^{\varepsilon}\right)\right)^{2}}-\left(s-\xi_{\beta}^{\varepsilon}\right) .
$$

Noting that $\xi_{\beta}^{\varepsilon}+\eta / 4<\xi_{1}<s$, in view of the mean value theorem, there exists $\xi_{2} \in\left(\xi_{\beta}^{\varepsilon}, s\right)$ such that

$$
-s+\frac{1}{\left(1+v^{\varepsilon}(s)\right)^{2}}=\left(\frac{-2 \dot{v}^{\varepsilon}\left(\xi_{2}\right)}{\left(1+v^{\varepsilon}\left(\xi_{2}\right)\right)^{3}}-1\right)\left(s-\xi_{\beta}^{\varepsilon}\right)<-\frac{\eta}{4} .
$$

Combining (5.4) with (5.6), we deduce

$$
\dot{v}^{\varepsilon}(\xi) \leq \frac{v_{+}}{\varepsilon C} \exp \left(-\frac{\eta}{4 \varepsilon}\left(\xi-\xi_{1}\right)\right)
$$

which implies that $\dot{v}^{\varepsilon}(\xi) \rightarrow 0$ uniformly on $\xi>\xi_{\beta}+\eta$.

For any $\xi>\xi_{\beta}+\eta$, we have

$$
v_{+}-v^{\varepsilon}(\xi)=\int_{\xi}^{+\infty} \dot{v}^{\varepsilon}(s) d s .
$$

Obviously we can see that $\lim _{\varepsilon \rightarrow 0^{+}} v^{\varepsilon}(\xi)=v_{+}$uniformly on $\xi>\xi_{\beta}+\eta$ by virtue of (5.7). Similarly, we have $\lim _{\varepsilon \rightarrow 0^{+}} v^{\varepsilon}(\xi)=0$ uniformly on $\xi<\xi_{\beta}-\eta$.

Lemma 5.2. Let $\xi_{\alpha}$ and $\xi_{\beta}$ be defined as above. Then we have the relation

$$
\xi_{\alpha}=\xi_{\beta}=\frac{1}{1+v_{+}} .
$$


Proof. Let us take $\phi(\xi) \in C_{0}^{\infty}\left(\xi_{1}, \xi_{2}\right)$, where $\xi_{1}<\xi_{\beta}<\xi_{2}$. From (4.3), this yields

$$
\int_{\xi_{1}}^{\xi_{2}} \varepsilon v_{\xi \xi} \cdot \phi(\xi) d \xi=\int_{\xi_{1}}^{\xi_{2}}\left(-\xi v_{\xi}+\left(\frac{v}{1+v}\right)_{\xi}\right) \phi(\xi) d \xi
$$

namely,

$$
\int_{\xi_{1}}^{\xi_{2}} \varepsilon v \phi^{\prime \prime}(\xi) d \xi=\int_{\xi_{1}}^{\xi_{2}}\left(v\left(\xi \phi^{\prime}(\xi)+\phi(\xi)\right)-\frac{v}{1+v} \phi^{\prime}(\xi)\right) d \xi .
$$

Passing to the limit $\varepsilon \rightarrow 0$ in (5.11) and taking notice of $v_{-}=0$, it can be derived from Lemma 1 that

$$
\int_{\xi_{\beta}}^{\xi_{2}}\left(v_{+}\left(\xi \phi^{\prime}(\xi)+\phi(\xi)\right)-\frac{v_{+}}{1+v_{+}} \phi^{\prime}(\xi)\right) d \xi=0
$$

Therefore

$$
-\left(v_{+} \xi_{\beta}-\frac{v_{+}}{1+v_{+}}\right) \cdot \phi\left(\xi_{\beta}\right)=0
$$

which yields $\xi_{\beta}=\frac{1}{1+v_{+}}$for $\phi(\xi)$ is arbitrary.

Obviously, we have

$$
\xi_{\alpha}=\lim _{\varepsilon \rightarrow 0^{+}} \xi_{\alpha}^{\varepsilon}=\lim _{\varepsilon \rightarrow 0^{+}} \frac{1}{1+v^{\varepsilon}\left(\xi_{\alpha}^{\varepsilon}\right)} \geq \frac{1}{1+v_{+}}=\xi_{\beta} .
$$

If $\xi_{\alpha}>\xi_{\beta}$, then we have $\lim _{\varepsilon \rightarrow 0^{+}} v^{\varepsilon}\left(\xi_{\alpha}^{\varepsilon}\right)=v_{+}$from Lemma 5.1. On the other hand, it follows that $\lim _{\varepsilon \rightarrow 0^{+}} v^{\varepsilon}\left(\xi_{\alpha}^{\varepsilon}\right)<v_{+}$from (5.14). This is a contradiction, which implies that $\xi_{\alpha}=\xi_{\beta}$.

LEMma 5.3. For arbitrary $\eta>0$, we have

$$
\lim _{\varepsilon \rightarrow 0^{+}} w^{\varepsilon}(\xi)= \begin{cases}w_{-}, & \text {for } \xi<\xi_{\alpha}-\eta, \\ w_{+}, & \text {for } \xi>\xi_{\alpha}+\eta,\end{cases}
$$

uniformly in the above intervals.

Proof. Let us suppose that $w_{ \pm}>0$ without loss of generality; otherwise the conclusion can be drawn similarly. Take $\varepsilon>0$ sufficiently small such that $\left|\xi_{\alpha}^{\varepsilon}-\xi_{\alpha}\right|<\eta / 2$ and $\left|\xi_{\beta}^{\varepsilon}-\xi_{\beta}\right|<\eta / 2$. For any $\xi_{1} \leq \xi_{\alpha}-\eta$, we have $\xi_{1} \leq \xi_{\alpha}^{\varepsilon}-\eta / 2$ for $\varepsilon$ sufficiently small.

Integration of (4.13) over $\left(-\infty, \xi_{1}\right)$ gives

$$
w^{\varepsilon}\left(\xi_{1}\right)=w_{-} \exp \int_{-\infty}^{\xi_{1}} \frac{\dot{v}^{\varepsilon}(s)}{1+v^{\varepsilon}(s)-s\left(1+v^{\varepsilon}(s)\right)^{2}} d s .
$$

For $s \in\left(-\infty, \xi_{1}\right)$ and $0 \leq v^{\varepsilon}(s) \leq v_{+}$, there exists $\xi_{2} \in\left(-\infty, \xi_{1}\right)$ such that

$$
\begin{aligned}
1+v^{\varepsilon}(s)-s\left(1+v^{\varepsilon}(s)\right)^{2} & =\left(1+v^{\varepsilon}(s)\right)^{2}\left(\frac{1}{1+v^{\varepsilon}(s)}-s-\frac{1}{1+v^{\varepsilon}\left(\xi_{\alpha}^{\varepsilon}\right)}+\xi_{\alpha}^{\varepsilon}\right) \\
& \geq\left(\frac{\dot{v}^{\varepsilon}\left(\xi_{2}\right)}{\left(1+v^{\varepsilon}\left(\xi_{2}\right)\right)^{2}}+1\right) \cdot\left(\xi_{\alpha}^{\varepsilon}-s\right) \\
& \geq \eta / 2 .
\end{aligned}
$$


Thus, from (5.16), we conclude that

$$
w^{\varepsilon}\left(\xi_{1}\right) \leq w_{-} \exp \int_{-\infty}^{\xi_{1}} \frac{2 \dot{v}^{\varepsilon}(s)}{\eta} d s=w_{-} \exp \left(\frac{2 v^{\varepsilon}\left(\xi_{1}\right)}{\eta}\right) .
$$

According to Lemma 5.1, we know that $\lim _{\varepsilon \rightarrow 0^{+}} v^{\varepsilon}\left(\xi_{1}\right)=0$ uniformly for $\xi_{1}<\xi_{\beta}-\eta=$ $\xi_{\alpha}-\eta$. Thus, we can see that $w^{\varepsilon}(\xi) \leq w_{-}$for any $\xi<\xi_{\alpha}-\eta$. On the other hand, it is obvious to see that $w^{\varepsilon}(\xi)$ is monotonically increasing in the interval $\left(-\infty, \xi_{\alpha}\right)$, so we have $w^{\varepsilon}(\xi) \geq w_{-}$for any $\xi<\xi_{\alpha}-\eta$. Hence, we obtain $\lim _{\varepsilon \rightarrow 0^{+}} w^{\varepsilon}(\xi)=w_{-}$uniformly for $\xi<\xi_{\alpha}-\eta$.

Finally, for any $\xi_{3} \geq \xi_{\alpha}+\eta$, it is easy to see that $\xi_{3} \geq \xi_{\alpha}^{\varepsilon}+\eta / 2$ for sufficiently small $\varepsilon>0$. Integrating (4.13) from $\xi_{3}$ to $+\infty$ again, we have

$$
w^{\varepsilon}\left(\xi_{3}\right)=w_{+} \exp \int_{\xi_{3}}^{+\infty} \frac{\dot{v}^{\varepsilon}(s)}{s\left(1+v^{\varepsilon}(s)\right)^{2}-1-v^{\varepsilon}(s)} d s .
$$

Also for $s \in\left(\xi_{3},+\infty\right)$, we have $s\left(1+v^{\varepsilon}(s)\right)^{2}-1-v^{\varepsilon}(s) \geq \eta / 2$, which implies that

$$
w^{\varepsilon}\left(\xi_{3}\right) \leq w_{+} \exp \int_{\xi_{3}}^{+\infty} \frac{2 \dot{v}^{\varepsilon}(s)}{\eta} d s=w_{+} \exp \left(\frac{2\left(v_{+}-v^{\varepsilon}\left(\xi_{3}\right)\right)}{\eta}\right) .
$$

Obviously we have $\lim _{\varepsilon \rightarrow 0^{+}} v^{\varepsilon}\left(\xi_{3}\right)=v_{+}$uniformly for $\xi_{3}>\xi_{\beta}+\eta=\xi_{\alpha}+\eta$; thus it follows that $w^{\varepsilon}(\xi) \leq w_{+}$holds for any $\xi>\xi_{\alpha}+\eta$. On the other hand, $w^{\varepsilon}(\xi)$ is monotonically decreasing in the interval $\left(\xi_{\alpha},+\infty\right)$, so we have $w^{\varepsilon}(\xi) \geq w_{+}$for any $\xi>\xi_{\alpha}+\eta$. Hence, we obtain $\lim _{\varepsilon \rightarrow 0^{+}} w^{\varepsilon}(\xi)=w_{+}$uniformly for $\xi>\xi_{\alpha}+\eta$.

Theorem 5.4. Suppose that $v_{+}>v_{-}=0$ and that $\left(v^{\varepsilon}(\xi), w^{\varepsilon}(\xi)\right)$ is the solution of (4.1) and (4.2). Then the limit of $\left(v^{\varepsilon}(\xi), w^{\varepsilon}(\xi)\right)$ as $\varepsilon \rightarrow 0$ is (3.6) together with (3.7), which is exactly the corresponding Riemann solution of (1.1) and (1.2).

Proof. In order to prove this theorem, we should study the limit behavior of $w^{\varepsilon}(\xi)$ in the neighborhood of $\xi=\sigma=\frac{1}{1+v_{+}}$in detail. To accomplish this, let us take $\phi(\xi) \in C_{0}^{\infty}\left[\xi_{1}, \xi_{2}\right]$ with $\xi_{1}<\sigma<\xi_{2}$ such that $\phi(\xi)=\phi(\sigma)$ for $\xi$ in a neighborhood $U$ of $\xi=\sigma\left(\phi\right.$ is called a sloping test function in [28]). Notice that $\sigma=\xi_{\alpha}=\xi_{\beta}$. Thus we have $\xi_{\alpha}^{\varepsilon} \in U$ for sufficiently small $\varepsilon>0$.

It follows from (4.13) that

$$
\int_{\xi_{1}}^{\xi_{2}}\left(-\xi w_{\xi}^{\varepsilon}+\left(\frac{w^{\varepsilon}}{1+v^{\varepsilon}}\right)_{\xi}\right) \phi(\xi) d \xi=\int_{\xi_{1}}^{\xi_{2}}\left(w^{\varepsilon} \phi+w^{\varepsilon}\left(\xi-\frac{1}{1+v^{\varepsilon}}\right) \phi^{\prime}\right) d \xi=0 .
$$

Then for arbitrary $\alpha_{1}, \alpha_{2} \in U$ satisfying $\alpha_{1}<\sigma<\alpha_{2}$, we have

$$
\int_{\xi_{1}}^{\xi_{2}}\left(w^{\varepsilon}\left(\xi-\frac{1}{1+v^{\varepsilon}}\right) \phi^{\prime}\right) d \xi=\int_{\xi_{1}}^{\alpha_{1}}\left(w^{\varepsilon}\left(\xi-\frac{1}{1+v^{\varepsilon}}\right) \phi^{\prime}\right) d \xi+\int_{\alpha_{2}}^{\xi_{2}}\left(w^{\varepsilon}\left(\xi-\frac{1}{1+v^{\varepsilon}}\right) \phi^{\prime}\right) d \xi .
$$


According to Lemma 5.1 and Lemma 5.3, we have

$$
\begin{aligned}
& \lim _{\varepsilon \rightarrow 0^{+}} \int_{\xi_{1}}^{\xi_{2}}\left(w^{\varepsilon}\left(\xi-\frac{1}{1+v^{\varepsilon}}\right) \phi^{\prime}\right) d \xi \\
& =\int_{\xi_{1}}^{\alpha_{1}}\left(w_{-}(\xi-1) \phi^{\prime}\right) d \xi+\int_{\alpha_{2}}^{\xi_{2}}\left(w_{+}\left(\xi-\frac{1}{1+v_{+}}\right) \phi^{\prime}\right) d \xi \\
& =w_{-}\left(\alpha_{1}-1\right) \phi(\sigma)-w_{-} \int_{\xi_{1}}^{\alpha_{1}} \phi d \xi-w_{+}\left(\alpha_{2}-\frac{1}{1+v_{+}}\right) \phi(\sigma)-w_{+} \int_{\alpha_{2}}^{\xi_{2}} \phi d \xi .
\end{aligned}
$$

Letting $\alpha_{1} \rightarrow \sigma-$ and $\alpha_{2} \rightarrow \sigma+$ leads to

$$
\lim _{\varepsilon \rightarrow 0^{+}} \int_{\xi_{1}}^{\xi_{2}}\left(w^{\varepsilon}\left(\xi-\frac{1}{1+v^{\varepsilon}}\right) \phi^{\prime}\right) d \xi=\left(-\sigma[w]+\left[\frac{w}{1+v}\right]\right) \phi(\sigma)-\int_{\xi_{1}}^{\xi_{2}} H(\xi-\sigma) \phi(\xi) d \xi,
$$

in which

$$
H(\xi-\sigma)= \begin{cases}w_{-}, & \text {for } \xi<\sigma, \\ w_{+}, & \text {for } \xi>\sigma .\end{cases}
$$

Substituting (5.22) into (5.20), we have

$$
\lim _{\varepsilon \rightarrow 0^{+}} \int_{\xi_{1}}^{\xi_{2}}\left(w^{\varepsilon}-H(\xi-\sigma)\right) \phi(\xi) d \xi=\left(\sigma[w]-\left[\frac{w}{1+v}\right]\right) \phi(\sigma),
$$

for all sloping test functions $\phi(\xi) \in C_{0}^{\infty}\left[\xi_{1}, \xi_{2}\right]$.

For any $\psi(\xi) \in C_{0}\left[\xi_{1}, \xi_{2}\right]$, we can select a sloping test function $\phi(\xi)$ such that

$$
\phi(\sigma)=\psi(\sigma) \text { and } \max _{\xi \in\left[\xi_{1}, \xi_{2}\right]}|\psi(\xi)-\phi(\xi)|<\mu .
$$

Taking $w^{\varepsilon}(\xi) \in L^{1}\left[\xi_{1}, \xi_{2}\right]$ uniformly in mind, we have

$$
\lim _{\varepsilon \rightarrow 0^{+}} \int_{\xi_{1}}^{\xi_{2}}\left(w^{\varepsilon}-H(\xi-\sigma)\right) \psi(\xi) d \xi=\lim _{\varepsilon \rightarrow 0^{+}} \int_{\xi_{1}}^{\xi_{2}}\left(w^{\varepsilon}-H(\xi-\sigma)\right) \phi(\xi) d \xi+O(\mu) .
$$

Noting that $\phi(\sigma)=\psi(\sigma)$, substituting (5.23) into (5.24) and then passing to the limit $\mu \rightarrow 0$, we can prove that

$$
\lim _{\varepsilon \rightarrow 0^{+}} \int_{\xi_{1}}^{\xi_{2}}\left(w^{\varepsilon}-H(\xi-\sigma)\right) \psi(\xi) d \xi=\left(\sigma[w]-\left[\frac{w}{1+v}\right]\right) \psi(\sigma)
$$

holds for all $\psi(\xi) \in C_{0}\left[\xi_{1}, \xi_{2}\right]$.

Letting $\xi_{1} \rightarrow \sigma-$ and $\xi_{2} \rightarrow \sigma+$ in (5.25), we can obtain

$$
w(\xi)=\lim _{\varepsilon \rightarrow 0^{+}} w^{\varepsilon}(\xi)=H(\xi-\sigma)+\left(\sigma[w]-\left[\frac{w}{1+v}\right]\right) \delta(\xi-\sigma) .
$$

Thus $w^{\varepsilon}(\xi)$ converges in the weak star topology of $C_{0}^{\infty}(R)$ and the limit of $w^{\varepsilon}(\xi)$ is a step function plus a Dirac delta function with strength $\sigma[w]-\left[\frac{w}{1+v}\right]$ and is now no longer self-similar, which is identical with (3.6) together with (3.7) constructed in section 3. 
In the end, we need to determine the value of $v(\xi)$ at the discontinuity point $\xi=\sigma$. It can be derived from (4.13) that

$$
\int_{\xi_{1}}^{\xi_{2}} w \cdot\left(\phi+\xi \phi^{\prime}-\frac{\phi^{\prime}}{1+v}\right) d \xi=0
$$

holds for any $\phi(\xi) \in C_{0}^{\infty}\left[\xi_{1}, \xi_{2}\right]$ with $\xi_{1}<\sigma<\xi_{2}$.

Substituting (5.26) into (5.27) yields

$$
\int_{\xi_{1}}^{\xi_{2}}\left(H(\xi-\sigma)+\left(\sigma[w]-\left[\frac{w}{1+v}\right]\right) \delta(\xi-\sigma)\right) \cdot\left(\phi+\xi \phi^{\prime}-\frac{\phi^{\prime}}{1+v}\right) d \xi=0 ;
$$

namely

$$
\begin{aligned}
0= & \int_{\xi_{1}}^{\sigma-}\left(\phi+\xi \phi^{\prime}-\frac{\phi^{\prime}}{1+v}\right) d \xi+\int_{\sigma+}^{\xi_{2}}\left(\phi+\xi \phi^{\prime}-\frac{\phi^{\prime}}{1+v}\right) d \xi \\
& +\left(\sigma[w]-\left[\frac{w}{1+v}\right]\right)\left(\phi(\sigma)+\sigma \phi^{\prime}(\sigma)-\frac{\phi^{\prime}(\sigma)}{1+v_{\delta}}\right) .
\end{aligned}
$$

It follows from (5.29) that

$$
w_{-}(\sigma-1) \phi(\sigma)-w_{+}\left(\sigma-\frac{1}{1+v_{+}}\right) \phi(\sigma)+\left(\sigma[w]-\left[\frac{w}{1+v}\right]\right)\left(\phi(\sigma)+\sigma \phi^{\prime}(\sigma)-\frac{\phi^{\prime}(\sigma)}{1+v_{\delta}}\right)
$$

which implies that $v_{\delta}=v(\sigma)=v_{+}$for $\phi$ is arbitrary.

\section{REFERENCES}

[1] L.Ambrosio, Transport equation and Cauchy problem for $B V$ vector fields, Invent. Math., 158(2004), 227-260. MR2096794 (2005f:35127)

[2] L.Ambrosio, G.Crippa, A.Figalli and L.A.Spinolo, Some new well-posedness results for continuity and transport equations, and applications to the chromatography system, SIAM J. Math. Anal., 41(2009), 1890-1920. MR2564199 (2010k:35295)

[3] S.Bianchini, Stability of $L^{\infty}$ solutions for hyperbolic systems with coinciding shocks and rarefactions, SIAM J. Math. Anal., 33(2001), 959-981. MR.1885292 (2002m:35139)

[4] F.Bouchut, On zero pressure gas dynamics, in: Advances in Kinetic Theory and Computing, Ser. Adv. Math. Appl. Sci., vol. 22, World Sci. Publishing, River Edge, NJ, 1994, 171-190. MR1323183 (96e:76107)

[5] A.Bressan and W.Shen, Uniqueness of discontinuous ODE and conservation laws, Nonlinear Anal. TMA, 34(1998), 637-652. MR.1634652 (99d:34005)

[6] G.Q.Chen and H.Liu, Formation of $\delta$-shocks and vacuum states in the vanishing pressure limit of solutions to the Euler equations for isentropic fluids, SIAM J. Math. Anal., 34 (2003), 925-938. MR 1969608 (2004a:76080)

[7] C.M.Dafermos, Solutions of the Riemann problem for a class of hyperbolic system of conservation laws by the viscosity method, Arch. Rational Mech. Anal., 52(1973), 1-9. MR0340837 (49:5587)

[8] C.M.Dafermos, Admissible wave fans in nonlinear hyperbolic systems, Arch. Rational Mech. Anal., 106(1989), 243-260. MR.981663 (90m:35124)

[9] C.M.Dafermos and R.Diperna, The Riemann problem for certain classes of hyperbolic systems of conservation laws, J. Differential Equations, 20(1976), 90-114. MR0404871 (53:8671)

[10] V.G.Danilov and V.M.Shelkovich, Delta-shock wave type solution of hyperbolic systems of conservation laws, Quart. Appl. Math., 63(2005), 401-427. MR2169026 (2006j:35158)

[11] W.E, Yu.G.Rykov and Ya.G. Sinai, Generalized variational principles, global weak solutions and behavior with random initial data for systems of conservation laws arising in adhesion particle dynamics, Comm. Math. Phys., 177 (1996), 349-380. MR1384139 (98a:82077) 
[12] G.Ercole, Delta-shock waves as self-similar viscosity limits, Quart. Appl. Math., 58(2000), 177-199. MR.1739044 (2000j:35187)

[13] B.T.Hayes and P.G.LeFloch, Measure solutions to a strictly hyperbolic system of conservation laws, Nonlinearity, 9 (1996), 1547-1563. MR.1419460 (98f:35096)

[14] J.Hu, A limiting viscosity approach to Riemann solutions containing delta-shock waves for nonstrictly hyperbolic conservation laws, Quart. Appl. Math., 55 (1997), 361-373. MR1447583 (98c:35109)

[15] F.Huang and Z.Wang, Well-posedness for pressureless flow, Comm. Math. Phys., 222 (2001), 117146. MR.1853866 (2002g:35142)

[16] B.L.Keyfitz and H.C.Kranzer, Spaces of weighted measures for conservaion laws with singular shock solutions, J. Differential Equations, 118(1995), 420-451. MR.1330835 (96b:35138)

[17] D.J. Korchinski, Solution of a Riemann problem for a system of conservation laws possessing no classical weak solution, Thesis, Adelphi University, 1977.

[18] J.Li and H.Yang, Delta-shocks as limits of vanishing viscosity for multidimensional zero-presure gas dynamics, Quart. Appl. Math., 59(2001), 315-342. MR.1827367 (2002b:76088)

[19] J.Li, T.Zhang and S.Yang, The Two-Dimensional Riemann Problem in Gas Dynamics, Pitman Monographs and Surveys in Pure and Applied Mathematics, 98, Longman Scientific and Technical, 1998. MR1697999 (2000d:76106)

[20] M.Mazzotti, Occurrence of a delta-shock in non-linear chromatography, 6th International Congress on Industrial and Applied Mathematics, Zurich, 2007.

[21] M.Mazzotti, Non-classical composition fronts in nonlinear chromatography - Delta-shock, Ind. Eng. Chem. Res. 48(2009), 7733-7752.

[22] M. Mazzotti, A. Tarafder, J. Cornel, F. Gritti, and G. Guiochon, Experimental evidence of a deltashock in nonlinear chromatography, J. Chromatography. A, 1217(2010), 2002-2012.

[23] E.Yu.Panov and V.M.Shelkovich, $\delta^{\prime}$-shock waves as a new type of solutions to system of conservation laws, J. Differential Equations, 228(2006), 49-86. MR.2254184 (2007f:35188)

[24] V.M.Shelkovich, Singular solutions of $\delta$ - and $\delta^{\prime}-$ shock wave types of systems of conservation laws, and transport and concentration processes, Russian Math. Surveys, 63(2008), 473-546. MR2479998

[25] W.Sheng and T.Zhang, The Riemann problem for the transportation equations in gas dynamics, Mem. Amer. Math. Soc., 137(N654)(1999), Amer. Math. Soc., Providence, RI. MR1466909 (99g:35109)

[26] M.Slemrod and A.E.Tzavaras, A limiting viscosity approach for the Riemann problem in isentropic gas dynamics, Indiana Univ. Math. J. 38(1989), 1047-1074. MR.1029688 (90m:35119)

[27] M.Sun, Interactions of elementary waves for the Aw-Rascle model, SIAM J. Appl. Math., 69(2009), 1542-1558. MR2487160

[28] D.Tan, T.Zhang and Y.Zheng, Delta-shock waves as limits of vanishing viscosity for hyperbolic systems of conservation laws, J. Differential Equations, 112(1994), 1-32. MR.1287550 (95g:35124)

[29] B.Temple, Systems of conservation laws with invariant submanifolds, Trans. Amer. Math. Soc., 280(1983), 781-795. MR716850 (84m:35080)

[30] A.E.Tzavaras, Wave interactions and variation estimates for self-similar zero-viscosity limits for hyperbolic systems of conservation laws, Arch. Rational Mech. Anal., 135(1996), 1-60. MR1414293 (97h:35149)

[31] H.Yang, Riemann problem for a class of coupled hyperbolic systems of conservation laws, J. Differential Equations, 159(1999), 447-484. MR1730728 (2000j:35184)

[32] Y.B.Zeldovich and A.D. Myshkis, Elements of Mathematical Physics: Medium consisting of noninteracting particles (in Russian), Nauka, Moscow, 1973. MR0351195 (50:3684) 